BULLETIN OF THE

AMERICAN MATHEMATICAL SOCIETY

Volume 78, Number 4, July 1972

\title{
THE SPECTRUM OF AN AUTOMORPHISM
}

\author{
BY STEPHEN SCHEINBERG ${ }^{1}$
}

Communicated by Felix Browder, December 29, 1971

In a series of articles H. Kamowitz and I investigated the nature of $\sigma(T)$, the spectrum of an arbitrary automorphism of an arbitrary semisimple commutative Banach algebra. This study was begun as a by-product of [1], in which we made the incidental observation that $\sigma(T)$ must meet $\{z:|z-1| \geqq 1\}$, unless $T=I$. The following is a summary of the known necessary conditions $(\mathrm{N})$ and the known sufficient conditions $(\mathrm{S})$ on $\sigma(T)$.

N1. If $T^{k}=I$ (some $k \geqq 1$ ), then $\sigma(T)=$ a union of subgroups of the group of $k$ th roots of $1,[2]$.

S1. Every possibility consistent with N1 can occur (direct sums of rotations).

N2. If $T^{k} \neq I($ all $k \geqq 1)$, then $\sigma(T) \supseteq$ the unit circle, [2].

S2. It is common that $\sigma(T)=$ the unit circle, but $\sigma(T)$ can be an annulus, [2].

N3. If $T^{k} \neq I$ (all $k \geqq 1$ ), then $\sigma(T)$ must be connected, [3].

S3. The set of $\sigma(T)$ 's is closed under the mapping $1 / z$, and if $U=\bigcup_{\alpha} \sigma\left(T_{\alpha}\right)$ is bounded away from 0 and $\infty$, then $\bar{U}$ is $\sigma(T)$ for some $T$. If $R$ is a bounded region such that $\{1<|z|<a\} \subseteq R \subseteq\{1<|z|\}$ and $\{1<|z|\}-R$ is a semigroup under multiplication, then $\bar{R}$ is $\sigma(T)$ for some $T$. The hypothesis that $R$ be connected may be weakened somewhat, [3].

The purpose of this note is to extend the set of constructions of [3] to include cases where $\sigma(T)$ is not the closure of its interior. The following theorem illustrates the technique of attaching a line segment to a region.

THEOREM. Let $\sigma=\{z: 1 \leqq|z| \leqq 2\} \cup\{z: 2 \leqq z \leqq 3\}$. Then there is a semisimple Banach algebra $A$ and an automorphism $T$ of $A$ such that $\sigma(T)=\sigma$.

Proof. In the outline which follows I have omitted several routine calculations. Let $A$ be the set of all functions which are bounded and analytic on $\{1<|z|<2\}$ and $C^{\infty}$ on $\{1.5 \leqq z \leqq 3\}$ and satisfy $\left|f^{(n)}(z)\right|$ $\leqq B \max \left(1, n !(\log n)^{n}\right)$ for some $B<\infty$, all $n \geqq 0$, and $1.5 \leqq z \leqq 3$. Define $p(f)=\sup \{|f(z)|: 1<|z|<2\}+\inf B$. It is clear that $p$ is a norm for $A$ and that $A$ is complete with respect to $p$.

AMS 1969 subject classifications. Primary 4655, 4650, 4630.

Key words and phrases. Semisimple commutative Banach algebra, algebra automorphism, spectrum, Hadamard product, quasi-analytic class.

${ }^{1}$ Supported in part by NSF Grant GP-25084. 
Define $f * g=\sum_{-\infty}^{\infty} a_{n} b_{n} z^{n}$, where $f=\sum a_{n} z^{n}$ and $g=\sum b_{n} z^{n}$. When $f$ and $g$ belong to $A, f * g$ is analytic on $1<|z|<4$ and

$$
f * g(z)=\frac{1}{2 \pi i} \int_{|w|=1} f(w) g\left(\frac{z}{w}\right) \frac{d w}{w} \text { for } 1<|z|<2 .
$$

It follows that $f * g \in A$ and $p(f * g) \leqq$ const $p(f) \cdot p(g)$. Then $\|f\|$ $=$ const $p(f)$ defines a Banach algebra norm on $A$.

The mapping $f \rightarrow a_{n}$ is a homomorphism of $A$ onto $C$ for each $n$. If $a_{n}=0$ for all $n$, then $f \equiv 0$ : this is obvious for $1<|z|<2$; for $1.5 \leqq z \leqq 3$ it is a consequence of Carleman's theorem on quasi-analytic classes [4, Chapter 1], since the $n$th root of $n !(\log n)^{n}$ is asymptotic to $(n / e) \log n$. Thus, $A$ is semisimple.

Because of the rapid growth of $n !(\log n)^{n}$, every function which is analytic on a neighborhood of $\sigma$ belongs to $A$. Furthermore, if $g$ is such a function and $f$ is arbitrary in $A$, then $g f \in A$ and $\|g f\| \leqq$ const $\|f\|$.

Define $T: A \rightarrow A$ by $T f(z)=z f(z)$. $T$ is an automorphism of $A$ and $\sigma(T) \supseteq \sigma$. If $\lambda \notin \sigma$, use $g=1 /(z-\lambda)$ in the preceding paragraph and we see that $\sigma(T)=\sigma$.

REMARK. The construction given above can be extended. As an illustration let us attach a new line segment to the old one. For example, let $\sigma^{\prime}=\sigma \cup\{z: z=3+i y, 0 \leqq y \leqq 1\}$. Define $A^{\prime}$ to be all functions which are bounded and analytic on $\{1<|z|<2\}, C^{\infty}$ on each interval $\{1.5 \leqq z \leqq 3\}$ and $\{3+i y: 0 \leqq y \leqq 1\}$ with $\left|f^{(n)}\right| \leqq B \max \left(1, n !(\log n)^{n}\right)$ on both intervals, and satisfying the Cauchy-Riemann condition $(\partial / \partial x)^{n} f$ $=\left(i^{-1} \partial / \partial y\right)^{n} f$ at $z=3$. The rest of the proof continues now with very slight changes. (Observe that the Cauchy-Riemann condition guarantees that any function analytic on a neighborhood of $\sigma^{\prime}$ will belong to $A^{\prime}$ and that any member of $A^{\prime}$ which is 0 on $\sigma$ will be 0 on $\sigma^{\prime}$.)

With the method of the theorem, disjoint domains can be connected by line segments, subject to the semigroup requirement of $S 3$, and these constructions may be combined with those of [3] and iterated to produce quite complicated $\sigma(T)$.

ACKNOWLEDGEMENT. I thank Y. Katznelson for suggesting that I try the notion of quasi-analyticity to obtain an annulus with an attached line segment.

\section{REFERENCES}

1. H. Kamowitz and S. Scheinberg, Derivations and automorphisms of $L^{1}(0,1)$, Trans. Amer. Math. Soc. 135 (1969), 415-427. MR 38 \#1533.

2. The spectrum of automorphisms of Banach algebras, J. Functional Analysis 4 (1969), 268-276. MR 40 \#3316. 
3. S. Scheinberg, Automorphisms of commutative Banach algebras, Problems in Analysis, Princeton Univ. Press, Princeton, N.J., 1971, pp. 319-323.

4. R. E. A. C. Paley and N. Wiener, Fourier transforms in the complex domain, Amer. Math. Soc. Colloq. Publ., vol. 19, Amer. Math. Soc., Providence, R.I., 1934.

Department of Mathematics, Stanford University, Stanford, California 94305

Current address: Department of Mathematics, University of California, Irvine, California 92664 